\title{
Diseño de cadena de abastecimiento bajo el concepto de logística inversa para el sector manufacturero de papel en la zona centro del Valle del Cauca.
}

\author{
Supply chain design under reverse logistic concept for paper manufacturing \\ sector in the middle zone of Valle del Cauca \\ Diego Leon Peña Orozco, Diana Fernanda Bolaños Carranza, Paola Fernanda Salcedo Peláez \\ Ingeniería Industrial, Universidad del Valle, Cali, Colombia \\ diego.l.pena@correounivalle.edu.co
}

\begin{abstract}
Resumen- Hoy en día la utilización de Productos Fuera de Uso (PFU) en la industria manufacturera de papel, ha tomado un mayor interés tanto en el ámbito de los negocios como en la investigación. Este fenómeno se ha dado gracias a la conciencia ambiental que se está estructurando en estas empresas, y también gracias al avance en la gestión de la cadena de suministro.

Para la correcta recolección de los PFU, se debe diseñar una cadena de abastecimiento inversa que sea capaz de administrar o gestionar adecuadamente la recolección de estos productos, a un costo logístico menor. Dentro del diseño de la cadena de abastecimiento se tendrán en cuenta todos los componentes necesarios para la recolección del papel, tales como la localización del centro de recuperación para lo cual es necesario contar con la información de tres plantas manufactureras de papel y de centros de reciclajes ubicados en la zona centro del Valle del Cauca.
\end{abstract}

Palabras clave- Productos fuera de uso, industria manufacturera, cadena de abastecimiento, reciclaje, logística inversa.

\begin{abstract}
Nowadays utilization of obsolete products (UOP) in manufacturing paper, has taken a great interest both in terms of business and research. This phenomenon has been due to environmental awareness is being structured in such companies, and also to advancement in supply chain management.

For a correct collection of UOP, It must design a reverse supply chain that is able to properly administer or manage the collection of these products, with a low cost. In supply chain design will have in consideration all components required for paper collection, such as recovery center location, taking necessary information about three paper manufacturing plants and recycling centers located in the middle zone of Valle del Cauca.
\end{abstract}

Key Word - Obsolete products, manufacturing industry, supply chain, recycling, reverse logictics.

\section{INTRODUCCIÓN}

El creciente interés en el reciclaje de productos y materiales es uno de los resultados de la concientización ambiental, que se ha presentado durante las últimas décadas. En los últimos años y en el ámbito de la gestión de la cadena de abastecimiento se ha venido presentando especial interés a los procesos de recuperación de productos que finalizan su vida y que son desechados por los consumidores (PFU, Productos fuera de uso), pero que aún poseen un valor que puede ser recuperado a través de su reutilización, reciclaje o refabricación. El tema del reciclaje es esencial en Colombia; las cifras señalan que en el país se genera aproximadamente 40.000 toneladas diarias de residuos sólidos; de esta cantidad, entre el $42 \%$ y $45 \%$ de los residuos que terminan en los rellenos sanitarios son materiales reciclables, y de estos solo se recupera aproximadamente el 5\%. El no reciclar impacta el medio ambiente, además de generar aumento de costos en energía, materia prima y productividad [1].

Este problema plantea la necesidad de gestionar los residuos, dejándolos de considerar como materiales que quedan inservibles después de haberlos consumido. Muchos de estos materiales "inservibles" pueden alcanzar un valor económico si, en lugar de llevarlos a un depósito final, son sometidos a un proceso de transformación o acondicionamiento que les permita incorporarse de nuevo al proceso productivo, bien sea de la propia empresa que lo genera o de otra. Estos materiales más que residuos deberían ser considerados como PFU.

Los principales problemas que se presentan a la hora de reciclar los PFU están asociados con la inadecuada planeación y administración de la cadena de abastecimiento: deficiencias 
operativas y la falta de tecnificación en los procesos de adecuación y transformación de materiales, dificultades en la separación en la fuente, la recolección y el transporte selectivo. El éxito de recuperación de estos PFU depende, no solo del producto a manejar, ni del sistema de recuperación empleado, ni con qué fin es recuperado, tampoco con su interacción con el flujo de retorno, ni los mercados destinos, entre otros, sino también de la cadena de abastecimiento a través de la cual se esté recuperando el producto. Para conseguir la recuperación de los PFU es necesario ampliar el significado de la cadena de abastecimiento para incluir el concepto de logística inversa.

En este sentido este trabajo presenta el diseño de una cadena de abastecimiento enfocada en la logística inversa para el sector manufacturero de papel, ubicada en la zona centro del Valle del Cauca, con el fin de gestionar de manera eficiente los flujos presentes de los centros de reciclaje en las distintas ciudades escogidas (Guacarí, Buga y Tuluá), la demanda generada por parte de las plantas y la posible capacidad del Centro de Recuperación. Para el diseño de la cadena es adecuada la aplicación de modelos de localización y de transporte para la ubicación estratégica del Centro de Recuperación.

\section{METODOLOGÍA}

Para el desarrollo metodológico se tomó como base el modelo del problema de localización discreta trabajado en [2]; donde se desea encontrar el número óptimo y capacidad de las bodegas a abrir, de tal forma que se minimicen los costos totales de logística, representados por los costos totales de transporte, los costos fijos de instalaciones y los costos de inventario. Este trabajo es realizado en un país y en una cadena de abastecimiento específica, sin embargo, se puede generalizar para otros países y cadenas de abastecimiento. Este modelo, a través de un análisis comparativo con la situación actual del caso de estudio se adapta para programar la operación de diseño de una cadena de abastecimiento bajo el concepto de logística inversa. El sector manufacturero de papel, a partir de un conjunto de materias primas recicladas y vírgenes, fabrican diferentes productos. El reciclaje de cartón y papel es cada día más complejo, ya que los cartones y papeles recuperados, como se ha dicho antes, retornan a la industria con todo tipo de materiales diferentes a celulosa (adhesivos sintéticos, plásticos, tintas, parafinas, metales etc.). Se desea realizar un diseño de una cadena de abastecimiento, con programación lineal entera mixta, partiendo de la oferta y demanda existente de los centros de reciclaje y plantas. Conocida la demanda de los materiales de las plantas y la disponibilidad de capacidad de los centros de reciclaje, se desea calcular: la localización del centro de recuperación de los PFU y la asignación de los correspondientes flujos de los PFU.
Para abordar el problema se plantea a continuación el modelo de referencia, el modelo propuesto y la validación que justifica la propuesta.

\section{A. Modelo de referencia}

Función objetivo: Minimizar costos totales de transporte y fijos

$$
\begin{gathered}
\sum_{i} \sum_{j} \text { FLETE_PROV }_{-} B O D_{i j} * X_{i j}+ \\
\sum_{j} \sum_{k} \text { FLETE_BOD_CON } S_{j k} * Y_{j k}+\sum_{j} \text { COSTOF }_{j} * \\
W_{j}
\end{gathered}
$$

Restricciones del modelo:

- Capacidad de proveedores

$$
\sum_{j} X_{i j} \leq \text { CAP_PROV }_{i} \forall i
$$

- Capacidad de bodegas (ilimitada para decidir capacidad)

$$
\sum_{i} X_{i j} \leq M W_{j} \forall j
$$

Donde $\mathrm{M}$ es un número positivo muy grande.

- Satisfacción de demanda de cada cliente

$$
\sum_{j} Y_{j k}=D E M A N D A_{k} \forall k
$$

- $\quad$ Balance de productos en bodegas

$$
\sum_{i} X_{i j}=\sum_{j} Y_{j k} \forall j
$$

- Número de bodegas a abrir

$$
\sum_{i} W_{i}=N
$$

\section{B. Programación de modelo propuesto}

\section{Conjuntos e índices}

CERE

$=$ Conjunto de Centros de reciclaje; indexada por $i$

CEREC

$=$ Conjunto de Centros de Recuperación; indexada por $j$

PLAN = Conjunto de plantas; indexada por $k$

$M P=$ Conjunto de Materias Primas; indexada por $l$

No existe un conjunto de modos de transporte, ya que este se va a analizar por vía terrestre a través de un tipo de vehículos estándar.

- Centros de reciclaje:

\begin{tabular}{|c|l|}
\hline \multirow{2}{*}{ GUACARÍ } & $\mathrm{A}=$ Centro de reciclaje 1 \\
\cline { 2 - 2 } & $\mathrm{B}=$ Centro de reciclaje 2 \\
\hline
\end{tabular}




\begin{tabular}{|c|c|}
\hline & $\mathrm{C}=$ Centro de reciclaje 3 \\
\hline \multirow{6}{*}{ BUGA } & $\mathrm{D}=$ Centro de reciclaje 4 \\
\hline & $\mathrm{E}=$ Centro de reciclaje 5 \\
\hline & $\mathrm{F}=$ Centro de reciclaje 6 \\
\hline & $\mathrm{G}=$ Centro de reciclaje 7 \\
\hline & $\mathrm{H}=$ Centro de reciclaje 8 \\
\hline & $\mathrm{I}=$ Centro de reciclaje 9 \\
\hline \multirow{5}{*}{ TULUÁ } & $\mathrm{J}=$ Centro de reciclaje 10 \\
\hline & $\mathrm{K}=$ Centro de reciclaje 11 \\
\hline & $\mathrm{L}=$ Centro de reciclaje 12 \\
\hline & $\mathrm{M}=$ Centro de reciclaje 13 \\
\hline & $\mathrm{N}=$ Centro de reciclaje 14 \\
\hline
\end{tabular}

Tabla 1. Información de los centros de reciclaje

\begin{tabular}{|l|l|}
\hline \multirow{3}{*}{$\begin{array}{c}\text { Posibles centros de } \\
\text { recuperación }\end{array}$} & $1=$ GUACARÍ \\
\cline { 2 - 2 } & $2=$ BUGA \\
\cline { 2 - 2 } & $3=$ TULUÁ \\
\hline
\end{tabular}

Tabla 2. Información sobre posibles centros de recuperación

\begin{tabular}{|l|l|}
\hline MPPL & Materia prima plegadiza (1) \\
\hline MPCA & Materia prima cartón (2) \\
\hline MPPE & Materia prima periódico (3) \\
\hline \multicolumn{2}{|c|}{ Tabla 3. Materias primas }
\end{tabular}

\begin{tabular}{|l|l|}
\hline PLAN1 & Planta número uno \\
\hline PLAN2 & Planta número dos \\
\hline PLAN3 & Planta número tres \\
\hline
\end{tabular}

Tabla 4. Plantas

\section{Parámetros}

CAP $_{\text {CERE } i l}=$ Capacidad de centros de reciclacje $i$ para materia prima recuperada $l\left(\frac{\text { ton }}{a \tilde{n} o}\right)$

$D E M_{k l}=$ demanda de la planta $k$

para materia prima recuperada l $\left(\frac{\text { ton }}{\text { año }}\right)$

COSTOF $_{j}=$ Costo fijo de un centro de recuperacion

$$
\text { en el lugar } j\left(\frac{\$}{a \tilde{n} o}\right)
$$

FLETE_CERE_CEREC $C_{i j}$

$=$ Flete entre el centro de reciclaje i y el centro

$$
\text { de recuperación } j\left(\frac{\$}{a \tilde{n} o}\right)
$$

$$
\text { FLETE_CEREC_PLAN }{ }_{j k}
$$

$=$ Flete entre el centro de recuperación $j$

$$
\text { y la planta } k\left(\frac{\$}{\text { ton }}\right)
$$

$M P_{l i j}=$ materia prima l entregada por el centro

de reciclaje i al centro de recuperación $j\left(\frac{\$}{a \tilde{n} o}\right)$
$M P_{l j k}=$ materia prima l recuperada por el centro

de recuperación j a la planta $k\left(\frac{\$}{a \tilde{n} o}\right)$

$\operatorname{RenMP} P_{l j k}$

$=$ Rendimiento de materia prima l recuperada por el centro de recuperación j a la planta $k\left(\frac{\$}{a \tilde{n} o}\right)$

$N=$ Número de centros de recuperación a abrir

\section{Variables de decisión}

$$
\begin{gathered}
X_{l i j}=\text { Cantidad de materia prima l a enviar } \\
\text { desde el centro de reciclaje } i \text { hacia } \\
\text { el centro de recuperación } j
\end{gathered}
$$

$Y_{l j k}=$ Cantidad de matria prima l a enviar desde el centro de recuperación $j$ hacia la planta $k$

$W_{j}$

$=1$ si se abre un nuevo centro de recuperación en el lugar $j$ y 0 de lo contrario

\section{Función objetivo}

Minimizar los costos totales $=$ costos de transporte + costos fijos de centro de recuperación

$$
\begin{aligned}
& \text { MinimizarCostosTotalesdeTransporteyFijo } \\
& =\sum_{i} \sum_{j} F_{L E T E_{C R E_{C E R E D} I J}} \\
& \text { * } \sum_{L} \sum_{I} \sum_{J} X_{l i j} \\
& +\sum_{j} \sum_{k} F L E T E_{-} C E R E C_{-} P L A N \\
& * \sum_{l} \sum_{i} \sum_{j} Y_{l i j} * \sum_{j} \operatorname{CosTOF}_{j} \\
& * W_{j}
\end{aligned}
$$

\section{Restricciones}

- Capacidad de los centros de reciclaje

$$
\sum_{j} X_{l i j} \leq C A P_{C E R E} ; \forall l, i
$$

Esta restricción expresa que todo el flujo de cada materia prima reciclada que sale de cada centro de reciclaje hacia los posibles centros de recuperación, no deberá ser mayor que su capacidad. La cantidad de restricciones está dada por el número de centros de reciclajes y materias primas recicladas.

- Capacidad de los centros de recuperación 


$$
\sum_{i} X_{l i j} \leq \sum_{k} D E M_{k l} W_{j} \forall j, l
$$

En esta restricción a diferencia del modelo de referencia, la capacidad del centro de recuperación estará limitada al valor total demandado de las plantas a las cuales se le va a servir (para el caso de que se abriera un solo centro de recuperación). Estas restricciones indican que todo el flujo que llega a cada centro de recuperación abierto desde los centros de reciclaje, no puede sobrepasar su capacidad. La cantidad de restricciones está dada por el número de ciudades potenciales para localizar el centro de recuperación y las materias primas recicladas.

- Demanda por producto de cada planta (para las tres plantas)

$$
\sum_{j} Y_{l i j} \leq D E M_{k l} \forall k, l
$$

$\checkmark \quad$ La Planta 1 demanda Materia Prima Periódico y Materia Prima Plegadiza.

$\checkmark \quad$ La Planta 2 demanda Materia Prima Plegadiza y Materia Prima Cartón.

$\checkmark \quad$ La Planta 3 demanda Materia Prima Plegadiza y Materia Prima Cartón.

Esta expresión responde al cumplimiento de la demanda de cada producto según la necesidad de cada una las tres plantas. Representa que la suma de los flujos que llegan desde todos los posibles centros de recuperación abiertos hacia cada planta, debe ser igual a su demanda estimada. En nuestro modelo esta expresión se hace por planta y por producto.

- Números de centros de recuperación

$$
\sum_{j} W_{j}=N
$$

Esta restricción expresa que el número total de centros de recuperación a abrir, es igual a la suma de las variables binarias asociadas a la apertura de cada centro de recuperación. Esta forma de la restricción se va a utilizar para que el modelo abra desde 1 hasta 3 bodegas, de tal forma que se pueda adicionar el costo de inventario respectivo.

- Balance de productos

$$
\begin{gathered}
\text { MPPL }=\text { Materia Prima Plegadiza Recuparada } \\
0.87 \sum_{i} X_{l i j}=\sum_{k} Y_{j k l} \forall j, l
\end{gathered}
$$

MPCAR = Materia Prima Carton Recuparada

$$
0.83 \sum_{i} X_{l i j}=\sum_{k} Y_{j k l} \forall j, l
$$

MPPER

= Materia Prima Periodico Recuparada

$$
0.83 \sum_{i} X_{l i j}=\sum_{k} Y_{j k l} \forall j, l
$$

Esta restricción indica que la cantidad de materia prima reciclada es igual a la cantidad de materia prima recuperada que sale hacia las diferentes plantas, teniendo en cuenta el rendimiento que generará después de la limpieza y adecuación que se realizará en el Centro de recuperación potencial; en esta expresión se encuentran 12 restricciones.

- Restricciones obvias

$$
\begin{gathered}
X_{i j}, Y_{j k} \geq 0 \forall i, j, k \\
W_{K} \in\{0.1\} \forall k
\end{gathered}
$$

Las restricciones obvias, expresan que los flujos de productos no pueden ser negativos y que las variables de apertura o cierre de los centros de recuperación son binarios.

\section{IMPLEMENTACIÓN DEL MODELO ESTABLECIDO.}

Con la información presentada en la situación actual, se implementó el modelo de programación lineal entera mixta propuesto en el lenguaje de modelado algebraico para programación matemática AMPL y utilizando como herramienta para la solución Neos Solver - Gurobi. Para el desarrollo del modelo se realizaron tres archivos, el primero corresponde al modelo de optimización, el segundo a los datos del modelo y el tercero a los comandos.

Durante el desarrollo del modelo de programación lineal entera mixta, se observó que el número de variables resultantes es de 156; de estas, 3 son variables binarias y 153 variables lineales. Así mismo se obtuvieron 61 restricciones.

\section{A. Resultados}

En esta sección se presenta los resultados obtenidos y los aspectos más importantes para el análisis. Los primeros respecto a la determinación de la localización del centro de recuperación y los flujos correspondientes.

\begin{tabular}{|c|c|}
\hline \multicolumn{2}{|c|}{ FUNCIÓN OBJETIVO } \\
\hline Costo total & $\$ 2.455 .320 .000$ \\
\hline \multicolumn{2}{|c|}{ Centros de recuperación a abrir } \\
\hline 1 & Guacarí \\
\hline 2 & Buga \\
\hline
\end{tabular}

Tabla 5. Función objetivo y localización centros de recuperación

Como se puede observar en la ¡Error! No se encuentra el origen de la referencia. anterior, la función objetivo indica que el valor mínimo total del costo fijo y de transporte es \$2.455.320.000 para dos centros de recuperación. En las siguientes tablas se presenta el flujo de los PFU desde cada 
centro de reciclaje hasta los centros de recuperación seleccionados.

\begin{tabular}{|c|c|c|c|}
\hline $\begin{array}{c}\text { FLUJO DE MATERIA PRIMA DESDE EL CENTRO DE } \\
\text { RECICLAJE HASTA EL CENTRO DE } \\
\text { RECUPERACIÓN 1 (TOn/año) }\end{array}$ \\
\hline $\begin{array}{c}\text { CENTROS } \\
\text { DE } \\
\text { RECICLAJE }\end{array}$ & \multicolumn{2}{c|}{ CENTRO DE RECUPERACIÓN 1 } \\
\cline { 2 - 4 } & CARTON & PERIODICO & PLEGADIZA \\
\hline A & - & - & - \\
\hline B & - & - & - \\
\hline C & - & - & - \\
\hline D & 370 & - & 300 \\
\hline E & 430 & - & 900 \\
\hline F & 560 & - & 1.400 \\
\hline G & - & 780 & 535 \\
\hline H & 540 & - & - \\
\hline I & 150 & - & 300 \\
\hline J & 120 & - & 450 \\
\hline K & - & - & - \\
\hline L & - & - & - \\
\hline M & - & 955 & 450 \\
\hline N & 330 & - & 300 \\
\hline TOTAL & $\mathbf{2 . 5 0 0}$ & $\mathbf{1 . 7 3 5}$ & $\mathbf{4 . 6 3 5}$ \\
\hline
\end{tabular}

Tabla 6. Flujo de los PFU desde el centro de reciclaje hasta el centro de recuperación 1 .

\begin{tabular}{|c|c|c|c|}
\hline $\begin{array}{c}\text { FLUJO DE MATERIA PRIMA DESDE EL CENTRO DE } \\
\text { RECICLAJE HASTA EL CENTRO DE } \\
\text { RECUPERACIÓN 2 (TOn/año) }\end{array}$ \\
\hline $\begin{array}{c}\text { CENTROS } \\
\text { DE } \\
\text { RECICLAJE }\end{array}$ & \multicolumn{3}{c|}{ CENTRO DE RECUPERACIÓN 2 } \\
\cline { 2 - 4 } & CARTON & PERIODICO & PLEGADIZA \\
\hline A & - & - & - \\
\hline B & - & - & - \\
\hline C & - & - & - \\
\hline D & - & 850 & - \\
\hline E & - & 833 & - \\
\hline F & - & 2.760 & - \\
\hline G & - & - & - \\
\hline H & - & 1.950 & 650 \\
\hline I & - & 445 & - \\
\hline J & - & 279 & - \\
\hline K & - & 1.080 & 922 \\
\hline L & - & 252 & - \\
\hline M & - & 450 & - \\
\hline N & - & 990 & - \\
\hline TOTAL & - & $\mathbf{9 . 8 8 9}$ & $\mathbf{1 . 5 7 2}$ \\
\hline
\end{tabular}

Tabla 7. Flujo de los PFU desde el centro de reciclaje hasta el centro de recuperación 2 .

\begin{tabular}{|c|c|}
\hline \multicolumn{2}{|c|}{$\begin{array}{c}\text { FLUJO DE MATERIA PRIMA DESDE EL CENTRO DE } \\
\text { RECICLAJE HASTA LOS CENTROS DE } \\
\text { RECUPERACIÓN (Ton/año) }\end{array}$} \\
\hline CENTROS & CENTROS DE RECUPERACIÓN \\
\hline
\end{tabular}

\begin{tabular}{|c|c|c|c|}
$\begin{array}{c}\text { DE } \\
\text { RECICLAJE }\end{array}$ & CARTON & PERIODICO & PLEGADIZA \\
\hline A & - & - & - \\
\hline B & - & - & - \\
\hline C & - & - & - \\
\hline D & 370 & 850 & 300 \\
\hline E & 430 & 833 & 900 \\
\hline F & 560 & 2.760 & 1.400 \\
\hline G & - & 780 & 535 \\
\hline H & 540 & 1.950 & 650 \\
\hline I & 150 & 445 & 300 \\
\hline J & 120 & 279 & 450 \\
\hline K & - & 1.080 & 922 \\
\hline L & - & 252 & - \\
\hline M & - & 1.405 & 450 \\
\hline N & 330 & 990 & 300 \\
\hline TOTAL & $\mathbf{2 . 5 0 0}$ & $\mathbf{1 1 . 6 2 4}$ & $\mathbf{6 . 2 0 7}$ \\
\hline
\end{tabular}

Tabla 8. Flujo de los PFU desde el centro de reciclaje hasta los centros de recuperación.

\section{DISTRIBUCIÓN DE LOS PFU}

घCENTRO DE RECUPERACIÓN 1 घ CENTRO DE RECUPERACIÓN 2

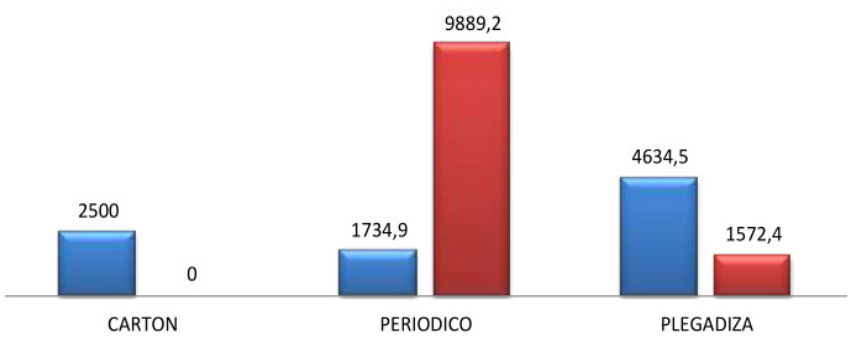

Ilustración 1. Representación de la distribución de los PFU.

Como se puede observar en el gráfico anterior, para el centro de recuperación 1 se acondicionarán 2.500 ton/año de Cartón, 1.734,9 Ton/año de Periódico y 4.634,5 ton/año de Plegadiza y para el centro de recuperación 2 se acondicionarán 9.889,2 ton/año de Periódico y $1.572,4$ ton/año de Plegadiza; es evidente que en este centro de recuperación no se almacenará Cartón, esto se debe a que este centro suplirá la demanda de la planta 1 ubicada en Buga, la cual no demanda Cartón para su proceso productivo. En la ¡Error! No se encuentra el origen de la referencia. se puede ver el flujo total de cada centro de reciclaje a los centros de recuperación; es claro que los PFU ofertados por los centros de reciclaje de la ciudad de Guacarí no están siendo utilizados por el sistema, esto es debido a que en estos centros se incurría en un costo de transporte, mientras que en los centros de reciclaje de Buga y Tuluá no, ya que estos centros llevan sus PFU a las plantas a las cuales les vende. Adicionalmente los demás centros de reciclajes poseen la capacidad requerida para suplir la demanda de las plantas escogidas en este modelo. 


\begin{tabular}{|c|c|c|c|c|c|c|c|c|c|}
\hline \multicolumn{7}{|c|}{$\begin{array}{l}\text { CENTROS DE RECUPERACIÓN } 1 \text { Y } 2 \text { HACIA LAS DIFERENTES } \\
\text { FLUJO DE MATERIA PRIMA DESDE (TON/ANO) }\end{array}$} \\
\hline $\begin{array}{c}\text { PLANTA 1 } \\
\begin{array}{c}\text { CENTROS DE } \\
\text { RECUPERACIÓN }\end{array}\end{array}$ & MPCA & MPPE & MPPL & MPCA & MPPE & MPPL & MPCA & MPPE & MPPL \\
\hline & & & & 2.400 & 600 & 1.512 & & 840 & 2.520 \\
\hline 1 Guacarí & & & PLANTA 2 & \multicolumn{3}{|c|}{ PLANTA 3 } \\
\hline 2 Buga & & 8.208 & 1.368 & & & & & & \\
\hline TOTAL & & $\mathbf{8 . 2 0 8}$ & $\mathbf{1 . 3 6 8}$ & $\mathbf{2 . 4 0 0}$ & $\mathbf{6 0 0}$ & $\mathbf{1 . 5 1 2}$ & & $\mathbf{8 4 0}$ & $\mathbf{2 . 5 2 0}$ \\
\hline
\end{tabular}

Tabla 9. Flujo de materia prima desde los centros de recuperación 1 y 2 hacia las plantas 1,2 y 3 .

El centro de recuperación 1 (Guacarí) suplirá la demanda de las plantas 2 y 3 ubicadas en la ciudad de Cali y el centro de recuperación 2 solo suplirá la demanda de la planta 1 ubicada en la ciudad de Buga. A continuación, se presenta la distribución de cada materia prima en las tres plantas.

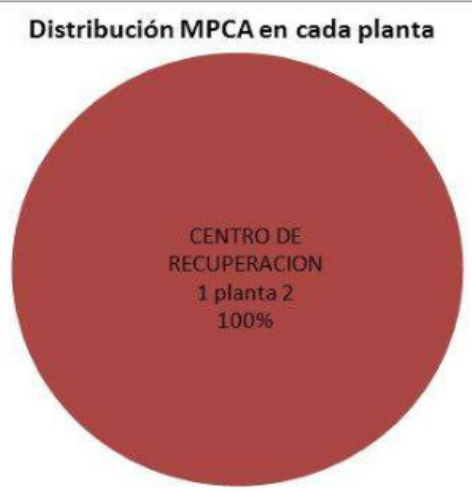

Ilustración 2. Distribución MPCA en cada planta.

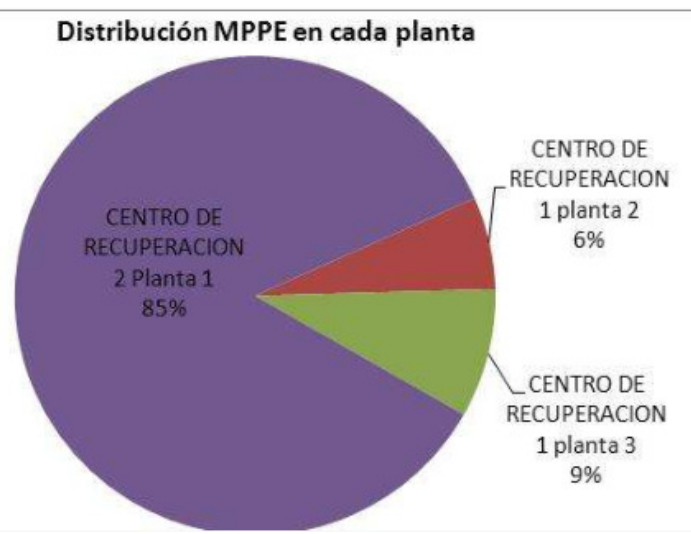

Ilustración 3. Distribución MPPE en cada planta.

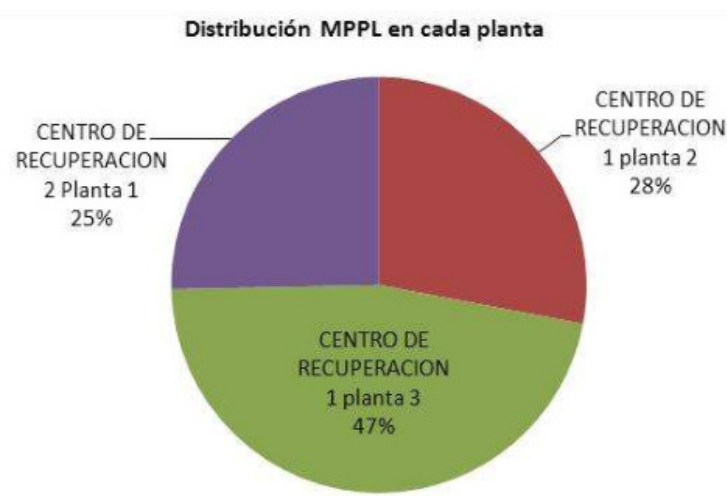

Ilustración 4. Distribución MPPL en cada planta.

Teniendo en cuenta los tres gráficos anteriores se puede observar que la planta 2 demanda toda la materia prima cartón que ofertan los centros de recuperación. Para la materia prima periódico las plantas 1,2 y 3 demandan de la siguiente manera $85 \%, 6 \%$, 9\% respectivamente; y para la materia prima plegadiza las plantas 1, 2 y 3 demandan el $25 \%, 28 \%$ y $47 \%$ respectivamente.

\section{B. Validación de resultados}

Con la ayuda del Excel se procede a validar los datos arrojados por el modelo de optimización y se corrobora la función objetivo. La función objetivo de este trabajo es la minimización de los costos de transporte y los costos fijos. El primer cuadro muestra los costos fijos de los centros de recuperación que se van abrir en los municipios de Guacarí y Buga.

\begin{tabular}{|c|c|}
\hline \multicolumn{2}{|c|}{ Costo Fijo de Centros de Recuperación Abrir } \\
\hline 1 & $\$ 48.000 .000$ \\
\hline 2 & $\$ 51.000 .000$ \\
\hline COSTO FIJO TOTAL & $\$ 99.000 .000$ \\
\hline
\end{tabular}

Tabla 10. Costos fijos de los centros de recuperación que van a operar.

La ¡Error! No se encuentra el origen de la referencia. relaciona los costos de transporte que se deben incurrir para llevar las diferentes materias primas de los centros de reciclaje a los centros de recuperación. 


\begin{tabular}{|c|c|c|c|}
\hline CERE A CEREC & FLETE & $\begin{array}{l}\text { CANTIDAD DE MP A ENVIAR DE } \\
\text { LOS CERE A LOS CEREC (ton/año) }\end{array}$ & \begin{tabular}{|c|} 
COSTO DE TRANSPORTE \\
DEL CERE A CEREC
\end{tabular} \\
\hline Flete del CR - A a 1. & $\$ 10.000$ & - & $\$ 0$ \\
\hline Flete del CR - B a 1. & $\$ 10.000$ & - & $\$ 0$ \\
\hline Flete del CR - C a 1. & $\$ 10.000$ & - & $\$ 0$ \\
\hline Flete del CR - A a 2. & $\$ 93.500$ & - & $\$ 0$ \\
\hline Flete del CR - B a 2. & $\$ 93.500$ & - & $\$ 0$ \\
\hline Flete del CR - C a 2. & $\$ 93.500$ & - & $\$ 0$ \\
\hline Flete del CR - D a 1. & $\$ 0$ & 670 & $\$ 0$ \\
\hline Flete del CR - E a 1. & $\$ 0$ & 1.330 & $\$ 0$ \\
\hline Flete del CR - F a 1. & $\$ 0$ & 1.960 & $\$ 0$ \\
\hline Flete del CR - G a 1. & $\$ 0$ & 1.315 & $\$ 0$ \\
\hline Flete del CR - Ha 1. & $\$ 0$ & 540 & $\$ 0$ \\
\hline Flete del CR - I a 1. & $\$ 0$ & 450 & $\$ 0$ \\
\hline Flete del CR - D a 2. & $\$ 0$ & 850 & $\$ 0$ \\
\hline Flete del CR - E a 2. & $\$ 0$ & 833 & $\$ 0$ \\
\hline Flete del CR - F a 2. & $\$ 0$ & 2.760 & $\$ 0$ \\
\hline Flete del CR - G a 2. & $\$ 0$ & - & $\$ 0$ \\
\hline Flete del CR - $\mathrm{H}$ a 2. & $\$ 0$ & 2.600 & $\$ 0$ \\
\hline Flete del CR - I a 2. & $\$ 0$ & 445 & $\$ 0$ \\
\hline Flete del CR - J a 1. & $\$ 0$ & 570 & $\$ 0$ \\
\hline Flete del CR - K a 1. & $\$ 0$ & - & $\$ 0$ \\
\hline Flete del CR - La 1. & $\$ 0$ & - & $\$ 0$ \\
\hline Flete del CR - Ma 1. & $\$ 0$ & 1.405 & $\$ 0$ \\
\hline Flete del CR - $\mathrm{N}$ a 1. & $\$ 0$ & 630 & $\$ 0$ \\
\hline Flete del CR - J a .2 & $\$ 0$ & 279 & $\$ 0$ \\
\hline Flete del CR - K a 2. & $\$ 0$ & 2.002 & $\$ 0$ \\
\hline Flete del CR - La 2. & $\$ 0$ & 252 & $\$ 0$ \\
\hline Flete del CR - Ma 2. & $\$ 0$ & 450 & $\$ 0$ \\
\hline Flete del CR - $\mathrm{N}$ a 2. & $\$ 0$ & 990 & $\$ 0$ \\
\hline
\end{tabular}

Tabla 11. Costos de Transporte de materias primas de los centros de reciclaje.

A continuación, se detallan los costos de transporte que se deben incurrir para llevar las diferentes materias primas de los centros de recuperación ubicadas en Guacarí y Buga a las diferentes plantas ubicadas en la zona centro del Valle del Cauca.

\begin{tabular}{|l|c|r|c|}
\hline CEREC A PLANTAS & FLETE & $\begin{array}{c}\text { CANTIDAD DE MP A ENVIAR DE LOS CEREC A } \\
\text { LAS PLANTAS (ton/año) }\end{array}$ & $\begin{array}{c}\text { COSTO DE } \\
\text { TRANSPORTE DEL }\end{array}$ \\
\hline CEREC 1 Planta 1 & $\$ 94.000$ & - & $\$ 0$ \\
\hline CEREC 1 Planta 2 & $\$ 275.000$ & 4.512 & $\$ 1.240 .800 .000$ \\
\hline CEREC 1 Planta 3 & $\$ 275.000$ & 3.360 & $\$ 924.000 .000$ \\
\hline CEREC 2 Planta 1 & $\$ 20.000$ & 9.576 & $\$ 191.520 .000$ \\
\hline CEREC 2 Planta 2 & $\$ 350.000$ & - & $\$ 0$ \\
\hline CEREC 2 Planta 3 & $\$ 350.000$ & - & $\$ 0$ \\
\hline \multicolumn{4}{|c|}{ COSTO TOTAL DE FLETE DE CEREC A PLANTAS } \\
\hline
\end{tabular}

Tabla 12. Costos de Transporte de materias primas de los Centros de recuperación a las plantas.

La validación a través de Excel de la Función Objetivo arroja los siguientes resultados, obtenidos de la suma de los costos de transporte tanto del envió de las materias primas de los centros de reciclaje a los centros de recuperación, como del centro de recuperación a las diferentes plantas más los costos fijos.

\begin{tabular}{|l|c|}
\hline \multicolumn{2}{|c|}{ FUNCIÓN OBJECTIVO } \\
\hline COSTO TOTAL DE FLETE DE CERE A CEREC & $\$ 0$ \\
\hline COSTO TOTAL DE FLETE DE CEREC A PLANTAS & $\$ 2.356 .320 .000$ \\
\hline COSTO FIJO TOTAL & $\$ \mathbf{9 9 . 0 0 0 . 0 0 0}$ \\
\hline COSTO TOTAL DE TRANSPORTE Y FIJO & $\mathbf{\$ 2 . 4 5 5 . 3 2 0 . 0 0 0}$ \\
\hline
\end{tabular}

Tabla 13. Costo total de transporte y costos fijos

Con este resultado se puede demostrar que la función objetivo arrojada por el modelo de optimización es la misma que la obtenida a través de la validación hecha en Excel.
A continuación, se presenta la validación del rendimiento de las materias primas manejadas en este modelo.

\begin{tabular}{|l|c|c|c|}
\hline & CARTON & PERIODICO & PLEGADIZA \\
\hline Demanda Total de las Plantas & 2.400 & 9.648 & 5.400 \\
\hline
\end{tabular}

Tabla 14. Cantidad de materia prima demandada por las plantas.

Es importante recordar que el rendimiento de cada materia prima en los centros de recuperación está dada de la siguiente manera: para el cartón se maneja un rendimiento de 0,96 , para el periódico 0,83 y para la plegadiza de 0,87 .

\begin{tabular}{|l|c|c|c}
\hline & CARTON & PERIODICO & PLEGADIZA \\
\hline Oferta de Total de los Centros de Recuperación & 2.500 & 11.624 & 6.207 \\
\hline Porcentaje de Rendimiento & 0,96 & 0,83 & 0,87 \\
\hline $\begin{array}{c}\text { TOTAL DE MP DESPUES DE LA LIMPIEZA Y } \\
\text { ACONTICIONAMIENTO }\end{array}$ & $\mathbf{2 . 4 0 0}$ & $\mathbf{9 . 6 4 8}$ & $\mathbf{5 . 4 0 0}$ \\
\hline
\end{tabular}

Tabla 15. Rendimiento de cada materia prima en los centros de recuperación.

De acuerdo con los resultados de la tabla anterior se puede confirmar que los resultados arrojados por el modelo, sí se aplicó el porcentaje de rendimiento de acuerdo a cada materia prima en cada centro de recuperación.

\section{CONCLUSIONES}

Las conclusiones más relevantes que se desprenden del trabajo realizado son las que se presentan a continuación:

- Con la determinación y descripción de las características del caso de estudio se logró identificar y plasmar en el modelo de optimización las diferentes restricciones de demanda, capacidad de centros de reciclaje y centros de recuperación, balanceo de productos y número de centro de recuperación a abrir.

- Se logra establecer la localización de los centros de recuperación y posterior asignación de flujos. El resultado obtenido por el modelo de programación arrojó la localización de los centros de recuperación en dos ciudades del centro del Valle del Cauca (Guacarí y Buga) y la asignación de los flujos desde los centros de reciclaje a los centros de recuperación y de estos a las plantas; teniendo como resultado la satisfacción de la demanda.

- El caso de estudio se realizó para un horizonte de un año, con 14 centros de reciclaje, 3 plantas y 3 tipos de PFU. Con esto, el resultado obtenido en la función objetivo es de $\$ 2.455 .320 .000$ anuales; de los cuales el $95,97 \%$ corresponden a transporte y el $4,03 \%$ a costos fijos. Con esto se evidencia que el mayor porcentaje dentro del costo total es el costo de transporte, debido a los fletes que se 
deben incurrir en el transporte de la materia prima demandada.

- El trazado de las rutas es un factor clave para conseguir mejores resultados en la minimización de distancia para la entrega de los productos fuera de uso de papel a las plantas manufactureras dentro de la cadena de abastecimiento.

- Para la factibilidad y cumplimiento de los objetivos planteados en el caso de estudio fue necesario incrementar la capacidad de los centros de reciclaje a su capacidad nominal, ya que se encontró que durante el desarrollo del modelo este presentaba un resultado de infactibilidad debido a que las capacidades de los centros de reciclajes tenían que ser mayores que la demanda de las plantas.
[9]. M. A. Ortega-Mier, Utilización de métodos cuantitativos para el análisis de problemas de localización en logística inversa., Madrid: Universidad Politécnica de Madrid, 2008.

[10]. J. Stock R. y D. Lambert M, Strategic Logistics Management, Estados Unidos, 2001.

A. d. Reciclaje, «Smurfut Kappa Cartón de Colombia S.A.,» (s.f). [En línea]. Available: www.smurfitkappa.com.co.

\section{REFERENCIAS}

[1]. [«Medio Ambiente Online,» 9 octubre 2008. [En línea]. Available: http://www.medioambienteonline.com/site/root/resou rces/industry_news/7153.

[2]. C. J. Vidal, Planeación, Optimización y Administración de Cadenas de Abastecimiento, Cali: Universidad del Valle, 2009.

[3]. R. Ballou, Logística: Administración de la Cadena de suministro., Mexico: Pearson Educación., 2004.

[4]. G. Clarke y W. Weight, Scheduling of vehicles from a central depot to a number of delivery points, Montevideo: Universidad de la República, 1964.

[5]. R. Dekker, M. Fleischmann, K. Inderfurth y L. Wassenhove, Reverse Logistics. Quantitative Models for Closed-Loop Supply Chains, Berlín, 2004.

[6]. «Mil toneladas de papel al mes recicla Comolsa para fabricar sus bandejas, estuches y empaques.,» El tiempo, 3 agosto 2010.

[7]. F. Kara, H. Rugrungruang y Kaebernick., «Simulation modelling of reverse logistics networks,» International Journal of Production Economics,, 2007.

[8]. A. Olivera, Heurísticas para Problemas de Ruteo de Vehículos, Montevideo: Universidad de la república, 2004. 